Mon. Not. R. Astron. Soc. 000, 田 (1998) Printed 22 August 2018 (MN LATEX style file v1.4)

\title{
The diffuse, relic radio source in Abell 85: estimation of cluster scale magnetic field from inverse Compton X-rays
}

\author{
J. Bagchi, ${ }^{1}$ V. Pislar, ${ }^{2}$ G.B. Lima Neto ${ }^{2}$ \\ 1 Indian Institute of Astrophysics, Koramangala, Bangalore 560 012, India \\ ${ }^{2}$ Institut d'Astrophysique de Paris, CNRS, 98bis Bd Arago, F-75014 Paris, France
}

Accepted ???. Received ????; in original form ????

\begin{abstract}
We report the first detection of an inverse Compton X-ray emission, spatially correlated with a very steep spectrum radio source (VSSRS), 0038-096, without any detected optical counterpart, in cluster Abell 85. The ROSAT PSPC data and its multiscale wavelet analysis reveal a large scale (linear diameter of the order of $500 h_{50}^{-1}$ $\mathrm{kpc}$ ), diffuse X-ray component, in excess to the thermal bremsstrahlung, overlapping an equally large scale VSSRS. The primeval $3 \mathrm{~K}$ background photons, scattering off the relativistic electrons can produce the X-rays at the detected level. The inverse Compton flux is estimated to be $(6.5 \pm 0.5) \times 10^{-13} \mathrm{erg} \mathrm{s}^{-1} \mathrm{~cm}^{-2}$ in the $0.5-2.4 \mathrm{keV} \mathrm{X}$ ray band. A new $327 \mathrm{MHz}$ radio map is presented for the cluster field. The synchrotron emission flux is estimated to be $(6.6 \pm 0.90) \times 10^{-14} \mathrm{erg} \mathrm{s}^{-1} \mathrm{~cm}^{-2}$ in the $10-100 \mathrm{MHz}$ radio band. The positive detection of both radio and X-ray emission from a common ensemble of relativistic electrons leads to an estimate of $(0.95 \pm 0.10) \times 10^{-6} \mathrm{G}$ for the cluster-scale magnetic field strength. The estimated field is free of the 'equipartition' conjecture, the distance, and the emission volume. Further, the radiative fluxes and the estimated magnetic field imply the presence of 'relic' (radiative lifetime $\gtrsim 10^{9} \mathrm{yr}$ ) relativistic electrons with Lorentz factors $\gamma \approx 700-1700$, that would be a significant source of radio emission in the hitherto unexplored frequency range $\nu \approx 2-10 \mathrm{MHz}$.
\end{abstract}

Key words: galaxies: clusters: Abell 85 - clusters: magnetic fields - clusters: X-rays - clusters: radio emission

\section{INTRODUCTION}

Apart from dark-matter, the diffuse intracluster medium has the two main constituents: the bremsstrahlung emitting hot $\left(T \sim 10^{7-8} \mathrm{~K}\right)$, tenuous $\left(n_{0} \sim 10^{-(3-4)} \mathrm{cm}^{-3}\right)$, and diffuse thermal gas, and the higher energy, relativistic particles (cosmic rays), with electrons emitting the magnetobremsstrahlung (synchrotron) radiation. The presence of diffuse, large scale $(0.2-1.0 \mathrm{Mpc})$ synchrotron sources with very steep spectra, is known in several clusters (e.g. Coma, Abell $2256,2319,85$, etc.). The origin of these relativistic particles is currently not well understood but in view of the absence of any optical counterparts, these radio sources are believed to be the remnants ('relics' and 'halos') of once active radio galaxies. These remnants are prevented from rapid fading from expansion by the thermal pressure of the surrounding intra cluster gas (Baldwin and Scott 1973). The main energy losses of their relativistic electrons come from synchrotron emission and the inverse Compton scattering of the $3 \mathrm{~K}$ background radiation. Their presence imply that magnetic fields on similar large scales may exist in the intracluster space (see Feretti \& Giovannini 1996, and Kronberg 1994 for reviews). That this magnetic field on large scales, may be a general property of clusters of galaxies, is suggested by the Faraday rotation data on radio sources observed in the direction of several clusters (Kim et al. 1991, Kronberg 1994). Estimates of the field strengths, based on Faraday rotation (Kim et al. 1991) or the 'equipartition' hypothesis (e.g. Feretti \& Giovannini 1996), both give a value $B \sim 0.5$ to $1.0 \mu \mathrm{G}$. Currently, the origin and evolution of these fields are not well understood due to observational difficulties in estimating the magnetic fields with sufficient accuracy and to the small number of actual estimates that has been attempted so far for a few clusters only (Kronberg 1994).

Another method of considerable merit to estimate the cluster scale magnetic field is the detection of co-spatial inverse Compton (IC) X-ray emission with the synchrotron emission plasma. The ubiquitous $3 \mathrm{~K}$ microwave background photons, scattering off the relativistic electrons (the $\mathrm{IC} / 3 \mathrm{~K}$ process), should produce a diffuse X-ray 'glow' associated 
with the radio plasma (Feenberg \& Primakoff 1948; Hoyle 1965; Baylis et al. 1967; Felten \& Morrison 1966; Harris \& Grindlay 1979; Rephaeli \& Gruber 1988). The nonrelativistic (thermal) analogue of this process is the well known Sunyaev-Zel'dovich effect (Rephaeli 1995). A possible detection of inverse Compton X-ray photons from the scattering of $3 \mathrm{~K}$ microwave background would not only provide a magnetic field estimate based on a different physical process, but would also provide information on as yet unknown non-thermal X-ray component in galaxy clusters. The inverse Compton method has several advantages: both the magnetic field strength, and the electron energy spectrum are obtainable from the observed $\mathrm{IC} / 3 \mathrm{~K}$ and the synchrotron emission fluxes, and the field so obtained is independent of the 'equipartition' conjecture (Pacholkzyck 1970), the distance, and the emission volume.

Despite a number of attempts over the last 20 years, the detection of $\mathrm{IC} / 3 \mathrm{~K}$ radiation has proved elusive, very often with only upper limits to the X-ray fluxes and lower limits to magnetic fields (e.g. Harris \& Romanishin 1974, Rephaeli 1977, Rephaeli et al. 1987, Rephaeli \& Gruber 1988, Bazzano et al. 1990, Rephaeli et al. 1994, Harris et al. 1995). The principal constraints arise from rarity of diffuse, steep spectrum cluster radio sources, limited sensitivity, and spatial/spectral resolution of low frequency (10-300 $\mathrm{MHz}$ ) radio or X-ray telescopes, and confusion from cluster thermal emission. However, correlating the radio and the EINSTEIN satellite data, Bagchi (1992) suggested the possibility of IC/3K emission in Abell 85. Recently, using the ROSAT PSPC data, Laurent-Muehleisen et al. (1994) and Feigelson et al. (1995) claimed the detection of IC/3K Xray emission associated with the radio lobes of the galaxy Fornax-A, further supported by Kaneda et al. (1995), employing the ASCA X-ray spectral data.

This work employs the good spatial resolution, high sensitivity and spectral capabilities of the ROSAT X-ray satellite, to search for co-spatial IC/3K emission from the diffuse radio source 0038-096 located in the rich cD cluster Abell 85. We present evidence for what might be the first detection of the IC/3K emission from the 'relic' relativistic electrons residing in the intra-cluster medium, presumably the remnants of an once active radio galaxy that is presently unidentified. We also present a new $327 \mathrm{MHz}$ radio map of the cluster field. Combining the radio and X-ray data, we estimate the magnetic field strength in the co-spatial emission volume. We then briefly discuss its implications. For a cluster redshift of 0.0555 (Pislar et al. 1997), 1 arcmin corresponds to $97 h_{50}^{-1} \mathrm{kpc}$, with the Hubble constant expressed in units of $50 \mathrm{~km} \mathrm{~s}^{-1} \mathrm{Mpc}^{-1}$.

\section{THE DATA}

\subsection{The OSRT Radio Observations}

Abell 85 was observed with the Ooty Synthesis Radio Telescope (OSRT; Sukumar et al. 1988), at a frequency of 326.5 $\mathrm{MHz}$ (band width $4 \mathrm{MHz}$ ), three times during 1985-86. After initial calibration, the visibility data were combined and 'self-calibration' (Schwab 1980) performed to further improve the quality. The calibrated data was Fourier transformed and the image was deconvolved with 'CLEAN' algorithm (using AIPS package), applied to the entire OSRT

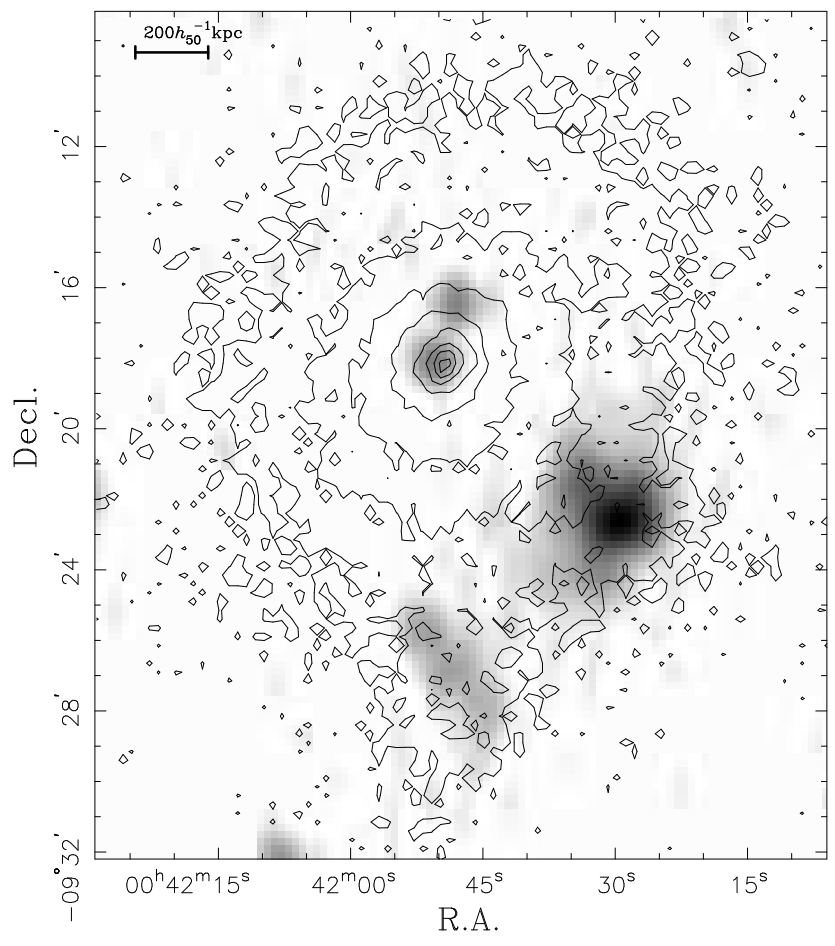

Figure 1. Iso-count contour map for the X-ray emission in Abell 85 (PSPC 0.1-2.4 keV data), superposed on OSRT $326.5 \mathrm{MHz}$ radio map (in grey scale). The contours are spaced logarithmically. The radio source 0038-096 is visible at $\mathrm{RA}(\mathrm{J} 2000) \sim 00^{\mathrm{h}} 41^{\mathrm{m}} 30^{\mathrm{s}}$, Decl. $\sim-09^{\circ} 23^{\prime}$. Note the excess X-ray emission at this location. Another correlated X-ray and radio emission could be seen over the 'south-blob' at RA $\sim 00^{\mathrm{h}} 41^{\mathrm{m}} 48^{\mathrm{s}}$, Decl. $\sim-09^{\circ} 27^{\prime} 30^{\prime \prime}$.

field of view ( $\sim 3 \mathrm{deg}$. EW x $0.75 \mathrm{deg} . \mathrm{NS})$. The final image was convolved with a 'clean' beam of 60 arcsec circular Gaussian (FWHM) profile. The $1-\sigma$ dispersion of noise on this image was $6.5 \mathrm{mJy} /$ beam area. This radio map is shown in Figs. 1 and 2. The OSRT has the necessary short baseline sampling to map the $\sim 5$ arcmin angular extent of the VSSRS.

\subsection{The ROSAT X-ray Observations}

The Abell 85 field was observed twice (PI Schwarz and PI Jones) by the ROSAT Position Sensitive Proportional Counter (PSPC). We have merged these two images to form one of effective exposure of $15949 \mathrm{~s}$ using the EXSAS package (Zimmermann et al. 1994). The iso-contours of this merged image are shown in Fig. 1. The PSPC has a point spread function (PSF) of $\approx 25 \operatorname{arcsec}(\mathrm{FWHM})$ within the central $\approx 10$ arcmin radius and at the $1 \mathrm{keV}$ energy.

We have processed the merged PSPC image using the multiscale wavelet reconstruction technique as described in Pislar et al. 1997 (and references therein). With the wavelet analysis, the noise is removed and we can detect structures (at different scales) significant at least at the 3 sigma level. The wavelet reconstruction of the PSPC data is shown in Fig. 2.

To obtain the X-ray spectrum of Abell 85 (which we will need for the calculation of the IC/3K emission, cf. below), we have used the EXSAS package, taking the background in a circle of radius 6 arcmin, without any visible sources, 31 


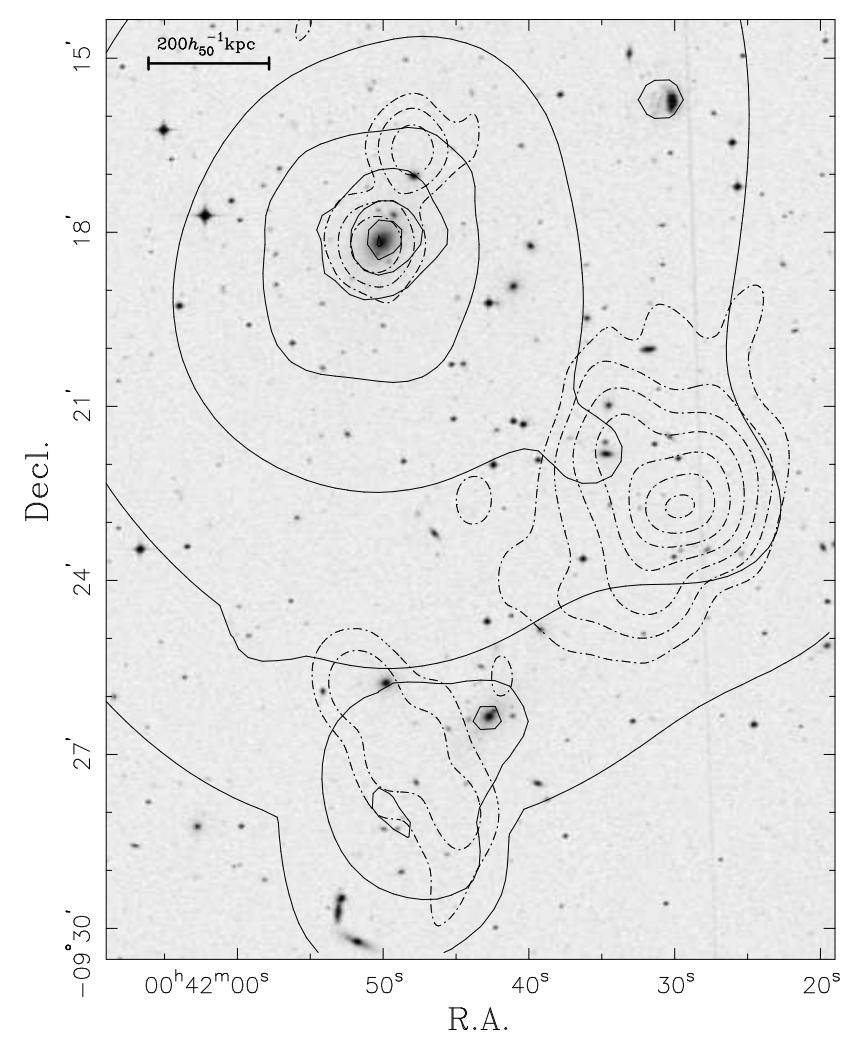

Figure 2. Abell 85 central region at different wavelengths. The photographic R-filter image (UK Schmidt Telescope and the Digitized Sky Survey) is shown in grey scale. Full contour lines show the multiscale wavelet reconstruction of the ROSAT PSPC Xray data shown in Fig. 1. The OSRT $326.5 \mathrm{MHz}$ radio surfacebrightness is depicted using dot-dashed contour lines. All contours are spaced logarithmically.

arcmin away from the centre of the cluster. The PSPC image was corrected for vignetting and the spectrum rebinned to obtain a signal to noise ratio of 5 .

\section{THE RADIO AND X-RAY STRUCTURES, AND THE FLUXES}

The correlated X-ray emission with the diffuse VSSRS at $\alpha=00^{\mathrm{h}} 41^{\mathrm{m}} 29^{\mathrm{s}} .5, \delta=-09^{\circ} 22^{\prime} 41^{\prime \prime}(\mathrm{J} 2000)$ can be seen in Fig. 1 (PSPC data) and Fig. 2 (wavelet reconstruction). The excess X-ray component is also diffuse, and has a similar large scale structure (about $500 h_{50}^{-1} \mathrm{kpc}$ across). The lack of optical identification (Fig. 2), and the extremely steep, curved radio spectrum of VSSRS (Fig. 3) suggest that it may be a 'relic' or 'halo' type source of undetermined origin, undergoing significant radiative losses (Slee \& Reynolds 1984, Swarup 1984, Joshi et al. 1986, Bagchi 1992). We note that the excess X-ray component can also be well discerned in the ROSAT HRI data (Lima Neto et al. 1997), and in the EINSTEIN observatory IPC image (Forman \& Jones 1982).

To estimate the IC/3K X-ray flux of VSSRS, we have carefully subtracted the thermal X-ray contribution from its vicinity using the detailed thermal bremsstrahlung model given by Pislar et al. (1997). From the model, we evaluated the parameters (ellipticity and position angle) of the elliptical iso-intensity contour passing over the VSSRS. Then

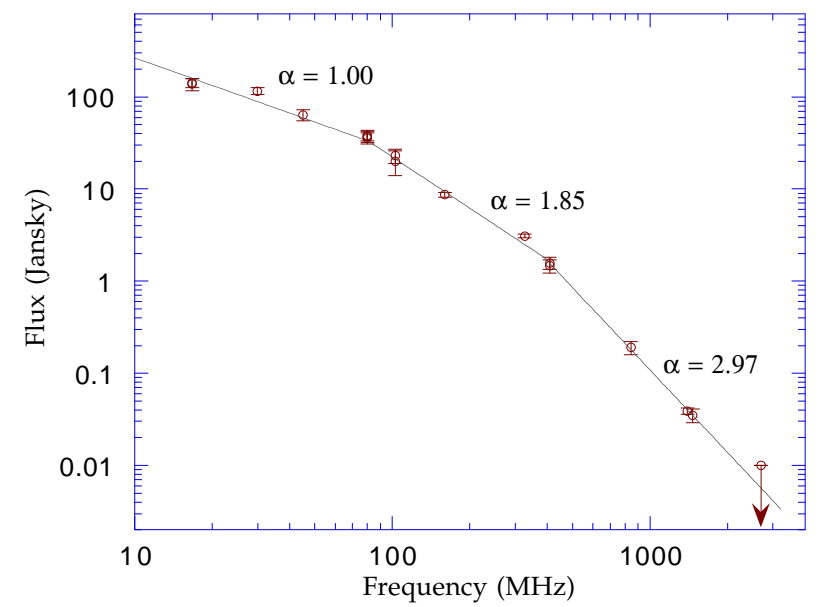

Figure 3. The radio spectrum of VSSRS 0038-096. The piecewise least-square spectral fits to the data are shown by three power laws with the spectral index $\alpha$ indicated alongside. The 326.5 $\mathrm{MHz}$ data point is the OSRT measurement $(3.15 \pm 0.15 \mathrm{Jy})$. Other data are from published literature.

using the actual PSPC counts, the bremsstrahlung flux was evaluated, using EXSAS, in 11 circular regions (excluding the VSSRS, the 'south blob', and the Seyfert galaxy at $\alpha$ $\left.(\mathrm{J} 2000)=00^{\mathrm{h}} 41^{\mathrm{m}} 30^{\mathrm{s}} \cdot 4, \delta=-09^{\circ} 15^{\prime} 48^{\prime \prime}\right)$, of 5 arcmin diameter each $(\approx$ the VSSRS diam.), placed on the isointensity contour. Optical spectroscopic analysis indicates the presence of a foreground group located towards the west of Abell 85 (Pislar et al. 1997); therefore we have proceeded in the following way: 7 of the 11 circles are taken on the north and east sides of the cluster, and the remaining 4 circles are located over the foreground group (where the counts are marginally higher). We have fixed the neutral hydrogen column density at each point at the galactic value (Dickey \& Lockman 1990).

After averaging the 11 measurements with weights appropriate to each (that is, depending on the region where it was taken), we obtained: $f_{t h}=(1.12 \pm 0.05) \times 10^{-12}$ erg $\mathrm{s}^{-1} \mathrm{~cm}^{-2}$, the thermal bremsstrahlung X-ray flux. We expressed the total flux over the VSSRS as the sum of $f_{t h}$, and the non-thermal IC/3K flux, $f_{I C}$. The $f_{I C}$ was obtained by fixing $f_{t h}$ at the above value and estimating $f_{I C}$ by a least-square fit to the total flux over the VSSRS. A priori, we have assumed that the $\mathrm{IC} / 3 \mathrm{~K}$ flux has the same spectral index $\alpha=1.00$ (flux $\propto \nu^{-\alpha}$ ) as the low frequency radio spectral index (Fig. 3), and have put the temperature of the bremsstrahlung emitting gas at the mean value of the 11 circular regions $(T=2.8 \mathrm{keV})$. This results in $f_{I C}=(6.5 \pm 0.5) \times 10^{-13} \mathrm{erg} \mathrm{s}^{-1} \mathrm{~cm}^{-2}$ in the $0.5-2.4 \mathrm{keV}$ band. The formal significance of $f_{I C}$ detection is about $9 \sigma$ (random errors).

The radio spectrum of VSSRS 0038-096 is shown in Fig. 3. For the present work, the flux density and the spectral index at about $10 \mathrm{MHz}$ are important. This is because an electron with Lorentz factor $\gamma \approx 1500$ would scatter the average $\nu_{b g} \approx 1.6 \times 10^{11} \mathrm{~Hz}$ microwave background photon (near the peak of Planck distribution) into the $\sim 2 \mathrm{keV}\left(\nu_{x}=\right.$ $\left.(4 / 3) \nu_{b g} \gamma^{2}=4.8 \times 10^{17} \mathrm{~Hz}\right) \mathrm{X}$-ray band, and would produce the synchrotron emission at $\nu_{\text {syn }} \approx 10-20 \mathrm{MHz}$ in a magnetic field of $B \sim 1-2 \mu \mathrm{G}\left(\nu_{\text {syn }}=4.19 B \gamma^{2} \mathrm{~Hz}\right)$. Fortunately, the 
spectrum is known near this frequency range. A power-law of spectral index $\alpha=1.00 \pm 0.10$ gives a good fit to the VSSRS spectrum in the frequency range $10-100 \mathrm{MHz}$ (Fig. 3). The synchrotron flux, $f_{S}=(6.6 \pm 0.9) \times 10^{-14} \mathrm{erg} \mathrm{s}^{-1} \mathrm{~cm}^{-2}$, is obtained by integrating in the $10-100 \mathrm{MHz}$ frequency range.

\section{THE MAGNETIC FIELD ESTIMATE}

Consider a population of relativistic electrons emitting the synchrotron and the $\mathrm{IC} / 3 \mathrm{~K}$ radiation. Given a power-law photon emission spectrum of index $\alpha=1.00$ (flux $\propto \nu^{-\alpha}$ ), the energy spectrum of electrons is another power law of index $p=2 \alpha+1=3.00 ; \mathrm{d} N(E)=N_{0} E^{-p} \mathrm{~d} E$, where $N_{0}$ is the amplitude, and $\mathrm{d} N(E)$, the differential number density within energy $E$ to $E+\mathrm{d} E$ (Blumenthal and Gould, 1970). Following the exact theoretical derivations by Blumenthal and Gould (1970), the equations for synchrotron flux $f_{S}$, and the IC/3K flux $f_{I C}$ can be put in the following practical forms (in cgs units, for the indicated bandwidths, and $\alpha=$ 1.00 photon sp. index):

$$
\begin{aligned}
& f_{S}=1.64 \times 10^{-14} \frac{N_{0} V}{4 \pi D^{2}} B^{2} a(3) \quad(10-100 \mathrm{MHz}) \\
& f_{I C}=1.36 \times 10^{-29} \frac{N_{0} V}{4 \pi D^{2}} T^{4} b(3) \quad(0.5-2.4 \mathrm{keV}) \text {. }
\end{aligned}
$$

Here, $V$ is the emission volume, $D$, the source distance, $B$, the magnetic field strength (randomly oriented in the intracluster medium), and $T=2.877 \mathrm{~K}$, is the radiation temperature at redshift 0.0555 (from COBE measure of $\mathrm{T}=2.726 \mathrm{~K}$ at $\mathrm{z}=0$; Mather et al. 1994). The factors $a(3)=0.0742$, and $b(3)=11.54$ are derived in Blumenthal and Gould (1970). The field B was estimated from these equations by substituting the observed values of $f_{S}$ and $f_{I C}$ :

$$
B=2.97 \times 10^{-6}\left(\frac{f_{S}}{f_{I C}}\right)^{\frac{1}{2}} \mathrm{G}=(0.95 \pm 0.10) \times 10^{-6} \mathrm{G}
$$

This estimate of $B$ is free of the uncertain quantities $N_{0}$, $V$, and $D$, being proportional to the ratio $\left(f_{S} / f_{I C}\right)^{\frac{1}{2}}$. The ' $1 / 2$ ' power dependence ensures that the estimated $B$ value would be only weakly affected by the errors in the radio and $\mathrm{X}$-ray fluxes. Note that this field estimate is independent of the bandwidths chosen for the X-ray and radio photons, as long as their power law spectral slopes are equal.

\section{DISCUSSION AND CONCLUSIONS}

We have presented an evidence for correlated X-ray emission with the diffuse VSSRS in Abell 85. What are the possible emission processes (other than the $\mathrm{IC} / 3 \mathrm{~K}$ ) that can give rise to the excess X-ray emission? The possibility that the emission is either the extension of the synchrotron radiation to the $\sim 1 \mathrm{keV}$ X-ray band, or that arises from the synchrotron self Compton process (Rees 1967, Harris et al. 1994), can be ruled out from the observed very steep spectral shape for $\nu \geq 1 \mathrm{GHz}$, signifying a dearth of necessary high energy photons. Further possibilities that the emission is generated by an active galaxy or that it arises from radio-jet clustermedium interaction also appear remote due to the relaxed, 'halo' type morphology of the VSSRS, and the absence of any optical counterpart.
Recent X-ray imaging and optical spectroscopy of clusters have shown the presence of thermal X-ray emission associated with secondary substructures possibly in the process of gravitational merger (e.g. Briel et al. 1991, Mohr et al. 1993, Henry and Briel 1993, Burns et al. 1994). Significantly, the presence of radio-halo sources appears to be correlated with the evidence for recent mergers, very high gas temperatures $(7-14 \mathrm{keV})$, large velocity dispersions $\left(\approx 1300 \mathrm{~km} \mathrm{~s}^{-1}\right)$, and the absence of both cooling flows and a single dominant central galaxy (BM type II or III; Edge et al. 1992, Tribble 1993, Feretti and Giovannini 1996). It has been proposed that these data possibly indicate the heating of intracluster gas and the reaccelaration of cosmic ray particles with stochastic amplification of magnetic fields, powered by the energy released in the ongoing mergers (Tribble 1993). Can the excess X-ray emission seen with the VSSRS in A85 come from such a process?

Although imaging data alone can not resolve this question, it is interesting to note that in terms of its physical properties, unlike the other radio-halo host clusters such as the Coma, Abell 2163, 2218, 2255,2256, and 2319, the cluster Abell 85 contains a central dominant $\mathrm{cD}$ galaxy, a central cooling flow of about $100 \mathrm{M}_{\odot} \mathrm{yr}^{-1}$, and relatively 'cool' gas at a temperature of $\mathrm{T} \sim 4 \mathrm{keV}$ (Pislar et al. 1997; Lima Neto et al. 1997).

Durret et al. (1998) have detected a filamentary structure, visible both in optical and in X-rays, linking Abell 85 to the neighbouring cluster Abell 87. They present evidence for a possible merger of matter in this filament with the southern region of Abell 85, in the 'south-blob' region. Markevitch et al. (1998) detect an enhancement in the gas temperature in the same region, possibly related to the merger. The position of the VSSRS, however, is not located there as it is shifted about 3 arcmin $\left(300 h_{50}^{-1} \mathrm{kpc}\right)$ to the northwest from the supposed shocked region and has a cooler temperature for the thermal matter in its vicinity.

The resolution of the question of exact physical process behind the excess X-ray emission would require high spectral (and spatial) resolution imaging spectroscopy data. In the hard X-ray energy regime of $\approx 10-20 \mathrm{keV}$, the contribution from thermal bremsstrahlung would drop sharply, whereas the non-thermal inverse Compton flux would be visible as an extra component with a steep power law spectrum of spectral index $\alpha \approx 1$. Based on the data presented in this work, we predict the possible $\mathrm{IC} / 3 \mathrm{~K}$ flux of $\approx 2.8 \times 10^{-13}$ erg $\mathrm{s}^{-1} \mathrm{~cm}^{-2}$, and the thermal bremsstrahlung flux of $\approx$ $3.0 \times 10^{-13} \mathrm{erg} \mathrm{s}^{-1} \mathrm{~cm}^{-2}$ in the hard X-ray band of 5-10 $\mathrm{keV}$. These fluxes are well within the reach of the currently operative BeppoSAX telescope and the upcoming AXAF and the XMM missions. The nature of the other excess Xray and the steep spectrum radio emission detected over the 'south-blob' (cf. below) could possibly also be understood with the spectroscopic X-ray data.

With the evidence currently available to us, energetically the most feasible mechanism for the X-ray emission from the VSSRS appears to be the IC/3K process. From theory (e.g. Ginzburg 1989), the (total) energy loss ratio can be shown to be $\frac{f_{I C}}{f_{S}} \approx U_{\text {rad }} / \frac{B^{2}}{8 \pi}$, where $U_{\text {rad }} \approx 5 \times 10^{-13}$ erg $\mathrm{cm}^{-3}$ is the energy density of the cosmological blackbody radiation field, and $\frac{B^{2}}{8 \pi} \approx 4 \times 10^{-14} \mathrm{erg} \mathrm{cm}^{-3}$ is the observed magnetic energy density. Therefore, if the observed 
radiation is from $\mathrm{IC} / 3 \mathrm{~K}$ process, we expect $f_{I C} / f_{S} \approx 13$, which is comparable to the observed ratio $f_{I C} / f_{S} \approx 10$ (the difference is mainly attributed to the finite bandwidths of our data). If the excess X-rays are produced by the thermal bremsstrahlung process, it is difficult to understand why the observed ratio is comparable to the ratio expected if IC/3K were the emission mechanism. This again suggests that the detected X-rays are indeed a product of inverse Compton scattering. This data on ratio of fluxes has enabled us to obtain a model independent magnetic field value, $B=0.95 \pm 0.10 \mu \mathrm{G}$, for the diffuse emission volume, located at $\sim 700 h_{50}^{-1} \mathrm{kpc}$ from the cluster centre.

It is apparent (Fig. 2) that another diffuse X-ray excess (the 'south-blob') is located at $\alpha=00^{\mathrm{h}} 41.8^{\mathrm{m}}, \delta=$ $-09^{\mathrm{d}} 27.5^{\mathrm{m}}$. In the same region, two radio components, without any definite optical counterparts, are observed with the OSRT with $>20 \sigma$ signal (162 mJy: northern source, 148 mJy: southern source). A spectral index limit, $\alpha>2.3$, is obtained for each of them, based on their non-detection in the new VLA $1.4 \mathrm{GHz}$ sky-survey (Condon et al. in preparation) to the $\approx 5 \mathrm{mJy}$ flux density limit. The nature of this excess X-ray emission is currently not well understood (Lima Neto et al. 1997). Is it possible that a second IC/3K X-ray source is located here, co-spatial with yet another relic radio plasma? If true, this can provide another magnetic field estimate at $\approx 1 h_{50}^{-1} \mathrm{Mpc}$ from the cluster centre. Detailed, low-frequency $(<327 \mathrm{MHz})$ radio and X-ray spectral data are necessary to explore this possibility.

Finally, the estimated magnetic field and the observed radiative fluxes from the VSSRS imply the presence of relativistic electrons with Lorentz factors $\gamma \approx 700-1700$ (for 0.5-2.4 keV band, $\nu_{x} \propto \nu_{b g} \gamma^{2}$ ). The population of electrons in this energy range would in turn produce significant radio emission $(\sim 1400 \mathrm{Jy}$ at $2 \mathrm{MHz})$ in the presently unexplored frequency range $\nu_{\text {syn }} \approx 2-10 \mathrm{MHz}\left(\nu_{\text {syn }} \propto B \gamma^{2}\right)$. The radiative lifetime of such 'relic' electrons is $\gtrsim 10^{9} \mathrm{yr}$ (Harris \& Grindlay 1979), comparable to the time-scale for evolution of clusters. Although this frequency range is below the ionospheric cutoff, the future generation radio telescopes observing from above the earth's atmosphere or from the far side of the moon (Burns 1990), could make such measurements. Such low frequency radio data in association with sensitive X-ray data would prove invaluable in probing the physics of the intracluster media in large scale structures.

It is encouraging to note that the inverse Compton $\mathrm{X}$ ray technique has the great potential, not only in measuring the elusive intra-cluster magnetic fields, but also in probing the hitherto unexplored population of very old relativistic electrons residing in the diffuse intra-cluster space.

\section{ACKNOWLEDGMENTS}

We thank F. Durret and D. Gerbal for fruitful comments and discussion. G. Swarup, V.K. Kapahi, and late M.N. Joshi are greatfully thanked for the OSRT radio data.

\section{REFERENCES}

Bagchi J. 1992, Ph.D. Thesis, Dept. of Physics, Indian Institute of Science, Bangalore, India

Baldwin J.E., Scott P.F., 1973, MNRAS, 165, 259
Baylis W.E., Schmidt W.M., Luscher E., 1967, Zeit. fur Astr. 66, 271

Bazzano A., Fusco-Femiano R., Ubertini P., Perrotti F., Quadrini E., Court A.J., Dean A.J., Dipper N.A., Lewis R., Stephen J.B., 1990, ApJ 362, L51

Blumenthal G.R., Gould R.J., 1970, Rev. Mod. Phys., 42(2), 237

Briel U.G., Henry J.P., Schwarz R.A., Bohringer H., Ebeling H., Edge A.C., Hartner G.D., Schindler S., Trumper J., Voges W., 1991, A\&A 246, L10

Burns J.O., 1990, Low Frequency Astrophysics from Space (Lecture Notes in Physics 362, Springer-Verlag), 19

Burns J.O., Rhee G., Owen F.N., Pinkney J., 1994, ApJ 423, 94

Dickey J.M., Lockman F.J., 1990, ARA\&A 28, 215

Durret F., Forman W., Gerbal D., Jones C., Vikhlinin A., 1998, A\&A in press astro-ph/9802183

Edge A., Steward G.C., Fabian A.C., 1992, MNRAS 258, 177

Feenberg E., Primakoff H., 1948, Phys. Rev. 73, 449

Feigelson E.D., Laurent-Muehleisen S.A., Kollgaard R.I., Fomalont E.B., 1995, ApJ 449, L149

Felten J.E., Morrison P., 1966, ApJ 146, 686

Feretti L., Giovannini G., 1996, IAU Symp. 175 "Extragalactic Radio Sources", p. 333 (Ekers R., et al. eds.)

Forman W., Jones C., 1982, ARA\&A 20, 547

Ginzburg V.L., 1989, Application of Electrodynamics in Theoretical Physics and Astrophysics, p. 431 (N.Y.: Gordon \& Breach)

Goldshmidt O., Rephaeli Y., 1994, ApJ 431, 586

Harris D.E., Romanishin W., 1974, ApJ 188, 209

Harris D.E., Grindlay J.E., 1979, MNRAS 188, 25

Harris D.E., Carilli C.L., Perley R.A., 1994, Nature 367, 713

Harris D.E., Willis A.G., Dewdney P.E., Batty J., 1995, MNRAS 273,785

Henry J.P., Briel U.G., 1993, Adv. Space Res. 13, (12)191

Hoyle F., 1965, Phys. Rev. Lett. 15, 131

Joshi M.N., Kapahi V.K., Bagchi J., 1986, Proc. of NRAO Workshop on Radio Continuum Processes in Clusters of Galaxies, p. 73 (O'Dea C.P., Uson J.M eds.)

Kaneda H., Tashiro M., Ikebe Y., Ishisaki Y., Kubo H., Makishima K., Ohashi T., Saito Y., Tabara H., Takahashi T., 1995, ApJ 453, L16

Kim K.-T., Tribble P.C., Kronberg P.P., 1991, ApJ 379, 80

Kronberg P.P., 1994, Rep. Prog. Phys. 57, 325

Laurent-Muehleisen S.A., Feigelson E.D., Kollgaard R.I., Fomalont E.B., 1994, in: "The Soft X-Ray Cosmos",AIP Conference Proceedings 313, p. 418, (Schlegel E.M., Petre R. eds.)

Lima Neto G.B., Pislar V., Durret F., Gerbal D., Slezak E., 1997, A\&A 327,81

Mather J.C. et al., 1994, ApJ 420, 439

Markevitch M., Forman W.R., Sarazin C.L., Vikhlinin A., 1998, ApJ Submitted (astro-ph/9711289)

Mohr J.J., Fabricant D.G., Geller M.J., 1993, ApJ 413, 492

Pacholkzyk A.G., 1970, Radio Astrophysics, p. 171 (WH Freeman \& Co., San Francisco)

Pislar V., Durret F., Gerbal D., Lima Neto G.B., Slezak E., 1997, A\&A 322,53

Rees M.J., 1967, MNRAS 137, 429

Rephaeli Y., 1977, ApJ 212, 608

Rephaeli, Y., Gruber D.E., Rothschild R.E., 1987, ApJ 320, 139

Rephaeli Y., Gruber D.E., 1988, ApJ 333, 133

Rephaeli Y., Ulmer M., Gruber D.E., 1994, ApJ 429, 554

Rephaeli Y., 1995, ARA\&A 33, 541

Schwab F.R., 1980, Proc. SPIE 231, 18

Slee O.B., Reynolds J.E., 1984, Proc. Astr. Soc. Aust 5, 516

Sukumar S., Velusamy T., Rao P., Swarup G., Bagri D.S., Joshi M.N., Ananthkrishnan S., 1988, Bull. Astron. Soc. India 16, 93

Swarup G. 1984, JA\&A 5, 139

Tribble P.C., 1993, MNRAS 263, 31

Zimmermann H.U., Becker W., Belloni T., Döbereiner S., Izzo 
C., Kahabka P., Schwentker O., 1994, EXSAS Users' Guide, MPE Report 244

(C) 1998 RAS, MNRAS 000, 1 日 